\title{
Streptomyces tsukubensis sp. nov., a producer of the immunosuppressant tacrolimus
}

\author{
Hideyuki Muramatsu and Koji Nagai \\ The Journal of Antibiotics (2013) 66, 251-254; doi:10.1038/ja.2012.116; published online 9 January 2013
}

Keywords: Streptomyces tsukubensis; tacrolimus; FK-506

Streptomyces is well-known as a good producer of pharmaceutically important chemical compounds, not only antibiotics but also physiologically active compounds. Tacrolimus was found as a strong immunosuppressant from a culture broth of an actinomycete strain, 'Streptomyces tsukubaensis' $9993^{\mathrm{T}}$, which was isolated from a soil sample collected from Mount Tsukuba, Ibaraki, Japan, in 1984. The first report of this compound was published in 1987, ${ }^{1}$ and the drug was launched in 1993 in Japan as an immunosuppressive agent for the prevention of graft rejection after liver transplantation. To date, it has been sold in about 90 countries in several therapeutic areas, including organ transplantation, myasthenia gravis, articular rheumatism, lupus nephritis, ulcerative colitis and atopic dermatitis, among others.

After initial discovery of the compound, several taxonomically diverse streptomycete strains were reported as tacrolimus producers, including S. tacrolimicus ATCC 55098, 2 S. clavuligerus CKD $1119^{3}$ and S. kanamyceticus KCC S- $0433^{\mathrm{T}}$. Further, biosynthetic gene clusters of tacrolimus in some strains have been reported. ${ }^{5-7}$ To date, however, no effective taxonomic description of the species 'S. tsukubaensis' has been available. Clarification of the taxonomic position of strain $9993^{\mathrm{T}}$ is important not only for streptomycete taxonomy, but also for evolutionary studies of secondary metabolite biosynthesis gene clusters. We previously revealed that strain $9993^{\mathrm{T}}$ does not belong to any described species according to its $16 \mathrm{~S}$ rRNA gene sequence phylogeny. ${ }^{4}$ We therefore investigated its morphological and physiological characteristics and phylogeny to confirm its taxonomic position. Here, we report this taxonomic study of strain $9993^{\mathrm{T}}$ and propose $S$. tsukubensis sp. nov.

Strain $9993^{\mathrm{T}}$, the original strain of 'S. tsukubaensis', was isolated from a soil sample collected from Mount Tsukuba, Ibaraki, Japan in $1984,{ }^{1}$ and has been stored as a lyophilized ampule for 27 years. The suspension of lyophilized spores was inoculated on inorganic salts-starch agar (ISP medium 4) and incubated at $28^{\circ} \mathrm{C}$ for $1-2$ weeks. The culture was stored at $4{ }^{\circ} \mathrm{C}$ during this study. S. clavuligerus NBRC $13307^{\mathrm{T}}$ was obtained from Biological Resource Center, National Institute of Technology and Evaluation, Japan. Media preparations, morphological observations and carbon sources utilization tests were performed by the methods of Shirling and Gottlieb. ${ }^{8}$ For determination of morphological characteristics, the strain was incubated at $28^{\circ} \mathrm{C}$ for 14 days, then morphologically examined using light and scanning electron microscopy (Hitachi S-2600N, Hitachi High-Technologies Corp., Tokyo, Japan). Color determinations were referenced against the Methuen Handbook of Color (Methuen London Ltd., London, UK). Growth temperature was determined on ISP medium 4 at 5, 13, 15, 20, 25, 28, 30, 32, 34, 35, 36, 37 and $38^{\circ} \mathrm{C}$, while tolerance to sodium chloride was determined on yeast extract-malt extract agar (ISP medium 2) with $0,4,7,10$ and $13 \%$ of sodium chloride.

Amino acids in cell wall hydrolysates, cellular fatty acids, menaquinones and $\mathrm{G}+\mathrm{C}$ content of the genomic DNA and DNA-DNA reassociation value were determined at TechnoSuruga Laboratory Co., Ltd. (Shizuoka, Japan). Amino acids, menaquinones and $\mathrm{G}+\mathrm{C}$ content were analyzed by high-performance liquid chromatography. Cellular fatty acids were analyzed using the standard MIDI system. Whole-cell sugar composition was determined by Thin Layer Chromatography, following the method of Hasegawa et al. ${ }^{9}$ Polar lipids were analyzed by two-dimensional Thin Layer Chromatography method. ${ }^{10}$

The 16S rRNA gene sequence of the strain was determined in our previous study (Accession no.; AB217600). ${ }^{4}$ Homology search was performed using the FASTA program. ${ }^{11}$ DNA sequence data was downloaded from the NCBI web site (http://www.ncbi.nlm.nih.gov/). BLAST search ${ }^{12}$ was performed at the DDBJ web site (http:// www.ddbj.nig.ac.jp/). Validity of bacterial names was referenced against the List of Prokaryotic Names with Standing in Nomenclature (http://www.bacterio.cict.fr/). The phylogenetic trees were constructed with 68 closest valid species using the neighborjoining $^{13}$ or maximum-parsimony methods ${ }^{14}$ in CLUSTAL $\mathrm{X}^{15}$ or MEGA package. ${ }^{16}$

Strain $9993^{\mathrm{T}}$ grew well on ISP medium 4 and ISP medium 2 . The color of the substrate mycelium was pinkish white to grayish orange. The strain produced a grayish aerial mycelium abundantly on ISP medium 4, and moderately on ISP medium 2. It formed flexuous 
spore chains composed of $>10$ smooth surface spores (Figure 1). The shape of spores was cylindrical, $0.5-0.7 \mu \mathrm{m}$ in diameter and $0.7-0.8 \mu \mathrm{m}$ in length. It showed weak or poor growth on oatmeal agar (ISP medium 3), glycerol-asparagine agar (ISP medium 5), peptone-yeast extract-iron agar (ISP medium 6) and tyrosine agar (ISP medium 7). No or very few aerial mycelia were observed on these media.

Strain $9993^{\mathrm{T}}$ did not produce melanin or any other soluble pigments in the media tested. Growth occurred at $15-35^{\circ} \mathrm{C}$ and optimum growth was observed at $28^{\circ} \mathrm{C}$. No aerial mycelia were observed at $35^{\circ} \mathrm{C}$. Strain $9993^{\mathrm{T}}$ showed weak growth and different morphology on ISP medium 2 with $4 \%$ of sodium chloride from that with $0 \%$. After 1 -week incubation, the strain showed green colonies with a colorless edge, without aerial mycelium. Further, the colonies turned black in color after one more week of incubation, forming Micromonospora-like colonies. No growth was observed with $7 \%$. Other physiological characteristics are given in Table 1 and in the species description.

LL-Diaminopimelic acid, glycine, glutamic acid and alanine were detected in cell wall hydrolysates, and glucose was detected as wholecell sugar but mannose, ribose, rhamnose, galactose, arabinose, xylose and madurose were not. These results are typical of cell wall chemotype $\mathrm{I}^{17}$ The major cellular fatty acids were iso- $\mathrm{C}_{16: 0}$ $(21.67 \%)$, iso- $\mathrm{C}_{15: 0}$ (13.16\%), $\mathrm{C}_{16: 1}$ cis-9 (11.96\%) and $\mathrm{C}_{16: 0}$ (10.57\%). Phosphatidylethanolamine and diphosphatidylglycerol were detected as major phospholipids. The major menaquinones were MK-9 $\left(\mathrm{H}_{8}\right)(86.6 \%)$ and MK-9 $\left(\mathrm{H}_{6}\right)(8.0 \%)$. The DNA G+C content of strain $9993^{\mathrm{T}}$ was calculated to be $72.4 \mathrm{~mol} \%$. These chemotaxonomic characteristics of the strain agreed with those of the genus Streptomyces.

FASTA homology search result revealed that the closest species to strain $9993^{\mathrm{T}}$ was $S$. clavuligerus NBRC $13307^{\mathrm{T}}$ (Accession no.; AB184343). The 200 highest-scored sequences of the FASTA search were examined to exclude invalid species and redundancies. Finally, 45 species with similarity values to strain $9993^{\mathrm{T}}$ ranging from 98.38 to $97.16 \%$ were selected. Thirty-three species with similarity values ranging from 97.93 to $96.62 \%$ were selected from the 200 highestscored sequences of the BLAST search using same procedure. Ten species were common between the FASTA and BLAST results, so 68 sequences of valid species of the genus Streptomyces were used for further phylogenetic analysis. The neighbor-joining and maximum-parsimony phylogenetic trees on the basis of almostcomplete 16S rRNA gene sequences of strain $9993^{\mathrm{T}}$ and the selected 68 valid species indicated that strain $9993^{\mathrm{T}}$ did not form a reliable cluster with any valid species (Figure 2). The similarity value between strain $9993^{\mathrm{T}}$ and S. clavuligerus NBRC $13307^{\mathrm{T}}$ was $98.38 \%$. The

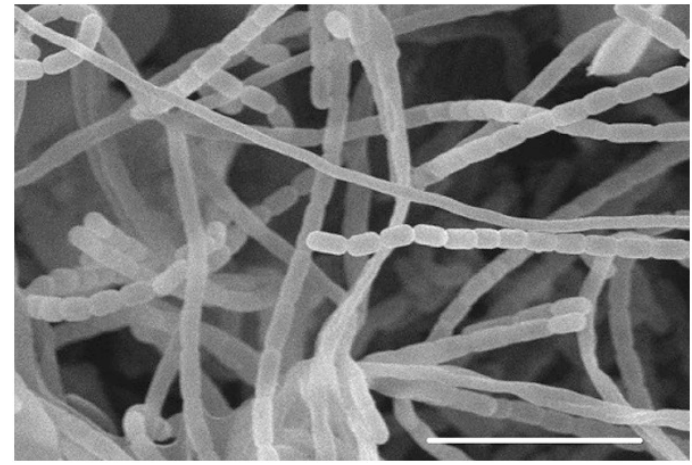

Figure 1 Scanning electron micrograph of strain $9993^{\top}$. Bar, $5 \mu \mathrm{m}$. average value of DNA-DNA reassociation between strain $9993^{\mathrm{T}}$ and NBRC $13307^{\mathrm{T}}$ was $12 \%$, which is consistent with Stackebrandt's theory. ${ }^{18}$ This result thus strongly indicates that strain $9993^{\mathrm{T}}$ does not belong to any known species. This conclusion was supported by differences in some phenotypic characters between strain $9993^{\mathrm{T}}$ and S. clavuligerus. ${ }^{19}$ S. clavuligerus had a club-shaped side-branching chain, which is uncommon in the genus Streptomyces, while strain $9993^{\mathrm{T}}$ had a branchless flexuous chain. Gelatin liquefaction was positive for strain $9993^{\mathrm{T}}$. These characteristics did not agree with those of S. clavuligerus. Utilization of dexran, D-fructose, galactose, lactose, D-melibiose and sodium propionate differed between strain $9993^{\mathrm{T}}$ and S. clavuligerus NBRC $13307^{\mathrm{T}}$ (Table 1).

Although 'S. tsukubaensis' NRRL 18488 is a patent strain, we consider that it is worthwhile to mention the relationship between strain $9993^{\mathrm{T}}$ and NRRL 18488 because the biosynthetic gene cluster for tacrolimus of NRRL 18488 is already available ${ }^{6}$ and the genome sequence project of NRRL 18488 is now ongoing. ${ }^{20}$ Strain $9993^{\mathrm{T}}$ was deposited on 19 October 1985 with the Fermentation Research Institute, Agency of Industrial Science and Technology (Japan) under the deposit number FERM BP-927 for patent application. ${ }^{21}$ According to another patent description, ${ }^{22}$ the strain FERM BP-927 was redeposited on 27 April 1989 with the Agricultural Research

\section{Table 1 Carbon source utilization of strain $9993^{\top}$ and S. clavuligerus NBRC $13307^{\top}$}

\begin{tabular}{lll}
\hline Carbon source & $9993^{T}$ & S. clavuligerus NBRC $13307^{T}$
\end{tabular}

\section{L-arabinose}

D-xylose

D-glucose

D-fructose

Sucrose

L-rhamnose

Raffinose

Inositol

D-Mannitol

Ribitol

D-cellobiose

Dextran

Dextrin

Dulcitol

Galactose

Glycerol

Inulin

-

Lactose

Mannose

Maltose

D-Melezitose

D-Melibiose

Salicin

Sorbitol

Starch

Sorbose

Trehalose

Xylitol

Sodium acetate

Sodium citrate

Sodium malonate

Sodium propionate

Sodium pyruvate

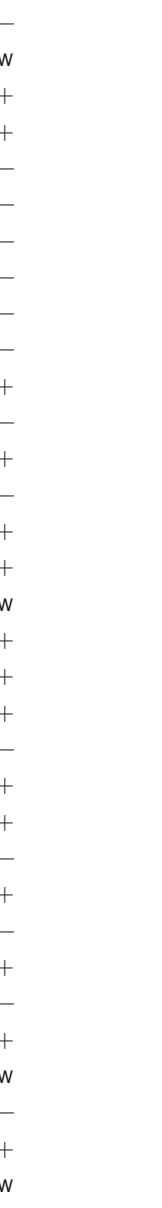

Abbreviations: +, positive; -, negative; w, weakly positive. 


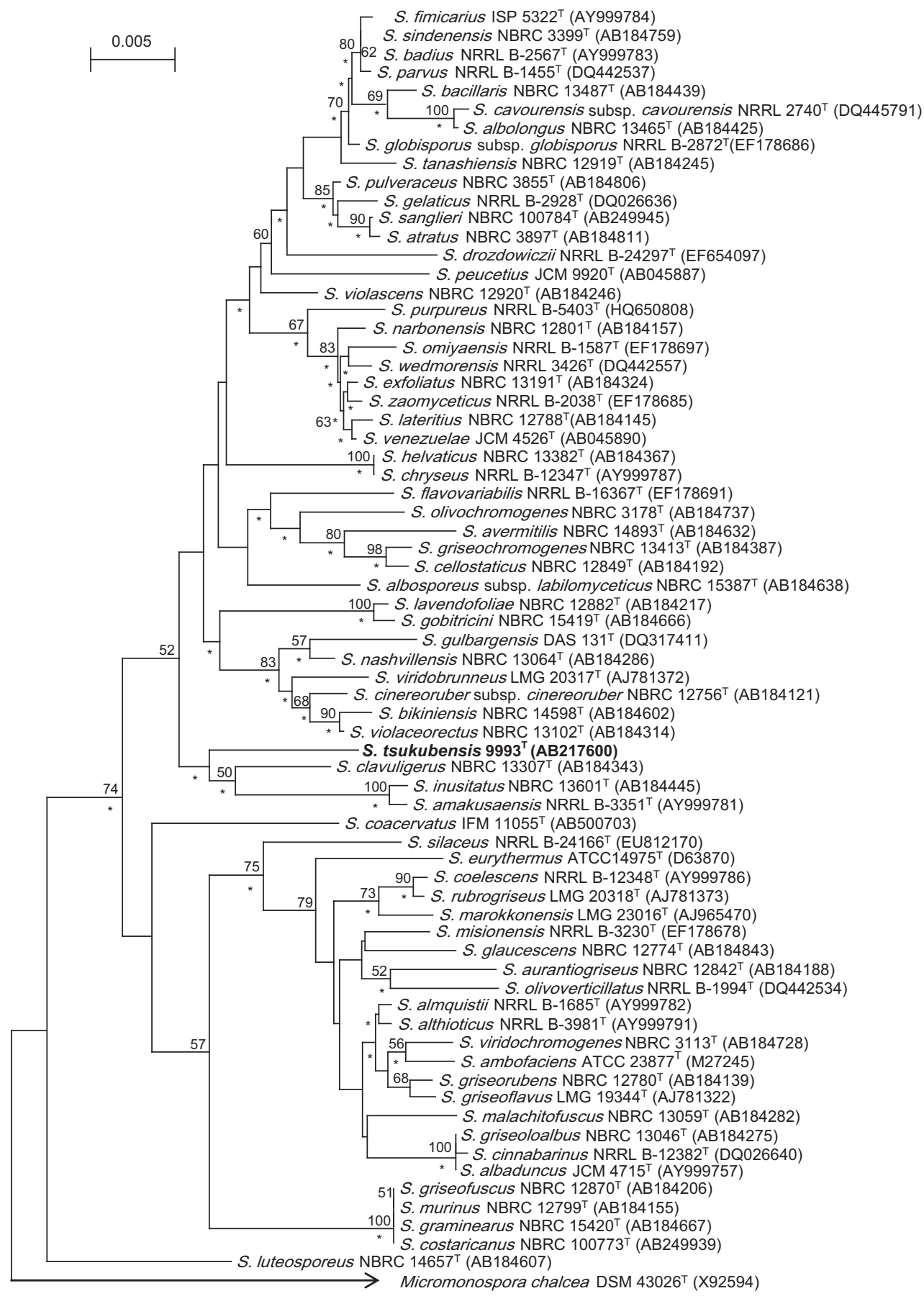

Figure 2 Neighbor-joining phylogenetic tree on the basis of almost-complete $16 \mathrm{~S}$ rRNA gene sequences showing the relationship between strain $9993^{\top}$ and closely related valid species of the genus Streptomyces. Bootstrap value $(>50 \%)$ on the basis of 1000 replicates are shown at branch nodes. Asterisks indicate branches on the tree that were also recovered with the maximum-parsimony method. Bar, 0.005 substitutions per nucleotide position.

Culture Collection International Depository (USA) under the deposit number NRRL 18488. Strain NRRL 18488 was therefore considered to be identical to strain $9993^{\mathrm{T}}$.

\section{Description of Streptomyces tsukubensis sp. nov.}

Streptomyces tsukubensis (tsu.ku.b.en'sis. N.L. masc. adj. tsukubensis pertaining to Mt. Tsukuba, Ibaraki, Japan, the origin of the soil sample from which the type strain was isolated).
A Gram-positive, aerobic actinomycete that forms extensively branched substrate hyphae. Vegetative hyphae are finely branched and do not fragment. Monopodially or dichotomously branching aerial mycelia develop abundantly. Grayish orange colonies are formed on ISP 2. Temperature range for growth is $15-35^{\circ} \mathrm{C}$, with optimum growth at $28^{\circ} \mathrm{C}$. D-Glucose, D-cellobiose, dextran, dextrin, glycerol, salicin and starch are used as sole carbon sources. Milk peptonization and gelatin liquefaction are positive, milk coagulation 
and cellulose decomposition are negative. Major menaquinones are MK-9 $\left(\mathrm{H}_{8}\right)$ and MK-9 $\left(\mathrm{H}_{6}\right)$. Major phospholipids are phosphatidylethanolamine and diphosphatidylglycerol. Major cellular fatty acids are iso- $\mathrm{C}_{16: 0}$, iso- $\mathrm{C}_{15: 0}, \mathrm{C}_{16: 1}$ cis-9 and $\mathrm{C}_{16: 0}$. The cell wall contains LL- $\mathrm{A}_{2} \mathrm{pm}$, glycine, glutamic acid and alanine. Contains glucose as a whole-cell sugar. The DNA $\mathrm{G}+\mathrm{C}$ content of the type strain is $72.4 \mathrm{~mol} \%$. The type strain, $9993^{\mathrm{T}}\left(=\mathrm{NBRC} 108819^{\mathrm{T}}\right)$, was isolated from soil from Mount Tsukuba, Japan, and produces the immunosuppressant tacrolimus.

\section{ACKNOWLEDGEMENTS}

We wish to thank Professor JP Euzeby for his advice of bacterial nomenclature. We also wish to thank Dr Morita Iwami, who isolated strain $9993^{\mathrm{T}}$, for encouraging us to publish this study, and Dr Takuya Wada for his technical support in the DNA sequence homology search.

1 Kino, T. et al. FK-506, a novel immunosuppressant isolated from a Streptomyces. I. Fermentation, isolation, and physico-chemical characteristics. J. Antibiot. 40 1249-1255 (1987).

2 Martínez-Castro, M., Barreiro, C., Romero, F., Fernández-Chimeno, R. I. \& Martín, J. F. Streptomyces tacrolimicus sp. nov. a low producer of the immunosuppressant tacrolimus (FK506). Int. J. Syst. Evol. Microbiol. 61, 1084-1088 (2011).

$3 \mathrm{Kim}, \mathrm{H}$. S. \& Park, Y. I. Isolation and identification of a novel microorganism producing the immunosuppressant tacrolimus. J. Biosci. Bioeng. 105, 418-421 (2008).

4 Muramatsu, H., Mokhtar, S. I., Katsuoka, M. \& Ezaki, M. Phylogenetic analysis of immunosuppressant FK506-producing streptomycete strains. Actinomycetologica 19, 33-39 (2005).

5 Motamedi, H. \& Shafiee, A. The biosynthetic gene cluster for the macrolactone ring of the immunosuppressant FK506. Eur. J. Biochem. 256, 528-534 (1998).

6 Goranovič, D. et al. Origin of the allyl group in FK506 biosynthesis. J. Biol. Chem. 285 14292-14300 (2010).
7 Mo, S. et al. Biosynthesis of the allylmalonyl-CoA extender unit for the FK506 polyketide synthase proceeds through a dedicated polyketide synthase and facilitates the mutasynthesis of analogues. J. Am. Chem. Soc. 133, 976-985 (2011).

8 Shirling, E. B. \& Gottlieb, D. Methods for characterization of Streptomyces species. Int. J. Syst. Bacteriol. 16, 313-340 (1966).

9 Hasegawa, T., Takizawa, M. \& Tanida, S. A rapid analysis for chemical grouping of aerobic actinomycetes. J. Gen. Appl. Microbiol. 29, 319-322 (1983).

10 Minnikin, D. E. et al. An integrated procedure for extracting bacterial isoprenoid quinones and polar lipids. J. Microbiol. Methods 2, 233-241 (1984).

11 Pearson, W. R. \& Lipman, D. J. Improved tools for biological sequence comparison. Proc. Natl Acad. Sci. USA 85, 2444-2448 (1988).

12 Altschul, S. F. et al. Gapped BLAST and PSI-BLAST: a new generation of protein database search programs. Nucleic Acids Res. 25, 3389-3402 (1997).

13 Saitou, N. \& Nei, M. The neighbor-joining method: a new method for reconstructing phylogenetic trees. Mol. Biol. Evol. 4, 406-425 (1987).

14 Eck, R. V. \& Dayhoff, M. O. Atlas of Protein Sequence and Structure (National Biomedical Research Foundation, Silver Spring, 1966).

15 Thompson, J. D., Gibson, T. J., Plewniak, F., Jeanmougin, F. \& Higgins, D. G. The Clustal_X windows interface: flexible strategies for multiple sequence alignment aided by quality analysis tools. Nucleic Acids Res. 25, 4876-4882 (1997).

16 Tamura, K. et al. MEGA5: molecular evolutionary genetics analysis using maximum likelihood, evolutionary distance, and maximum parsimony methods. Mol. Biol. Evol. 28, 2731-2739 (2011).

17 Lechevalier, M. P. \& Lechevalier, H. Chemical composition as a criterion in the classification of aerobic actinomycetes. Int. J. Syst. Bacteriol. 20, 435-443 (1970).

18 Stackebrandt, E. \& Ebers, J. Taxonomic parameters revisited: tarnished gold standards Microbiol. Today Nov., 152-155 (2006).

19 Higgens, C. E. \& Kastner, R. E. Streptomyces clavuligerus sp. nov., a $\beta$-lactam antibiotic producer. Int. J. Syst. Bacteriol. 21, 326-331 (1971).

20 Barreiro, C. et al. Draft genome of Streptomyces tsukubaensis NRRL 18488, the producer of the clinically important immunosuppressant tacrolimus. J. Bacteriol. 194, 3756-3757 (2012).

21 Okuhara, M., Tanaka, H., Goto, T., Kino, T. \& Hatanaka, H. Tricyclo compounds, process for their production and a pharmaceutical composition containing the same. US Patent 4,929,611 (1990).

22 Fehr, T. Substituted 4-azatricyclo (22.3.1.04.9) octacos-18-ene derivatives, their preparation and pharmaceutical compositions containing them. EP 0356399 A2 (1990). 